\title{
Téoros
}

Revue de recherche en tourisme

\section{Les processus de construction du géotourisme alpin}

\section{Nathalie Cayla}

Volume 29, numéro 2, 2010

\section{Géotourisme}

URI : https://id.erudit.org/iderudit/1024867ar

DOI : https://doi.org/10.7202/1024867ar

Aller au sommaire du numéro

Éditeur(s)

Université du Québec à Montréal

ISSN

0712-8657 (imprimé)

1923-2705 (numérique)

Découvrir la revue

Citer cet article

Cayla, N. (2010). Les processus de construction du géotourisme alpin. Téoros, 29(2), 15-25. https://doi.org/10.7202/1024867ar

\section{Résumé de l'article}

L'offre géotouristique proposée au sein de l'arc alpin par les six pays qui composent cette chaîne de montagne est abondante avec quelque 400 sites aménagés pour accueillir un public estimé à plus de cinq millions de visiteurs par an. Dans un premier temps, les processus de cette mise en tourisme sont étudiés au travers de deux filtres d'analyse : l'approche historique, l'émergence de cette offre plongeant ses racines dans les premières explorations des naturalistes voyageurs du XVI ${ }^{\mathrm{e}}$ siècle, mais également au regard des jeux d'acteurs qui sont à l'origine de ces réalisations. Loin de la standardisation de certaines pratiques touristiques, un panorama actuel de l'offre met en évidence une hétérogénéité importante dont l'étude permet de mieux cerner quelques spécificités du géotourisme. 


\title{
Les processus de construction du géotourisme alpin
}

\author{
Nathalie CAYLA \\ Docteure en sciences de la Terre, de l'univers et de l'environnement \\ Professeure agrégée, Laboratoire Edytem, Université de Savoie \\ Nathalie.cayla@univ-savoie.fr
}

\begin{abstract}
RÉSUMÉ: L'offre géotouristique proposée au sein de l'arc alpin par les six pays qui composent cette chaîne de montagne est abondante avec quelque 400 sites aménagés pour accueillir un public estimé à plus de cinq millions de visiteurs par an. Dans un premier temps, les processus de cette mise en tourisme sont étudiés au travers de deux filtres d'analyse : l'approche historique, l'émergence de cette offre plongeant ses racines dans les premières explorations des naturalistes voyageurs du XVle siècle, mais également au regard des jeux d'acteurs qui sont à l'origine de ces réalisations. Loin de la standardisation de certaines pratiques touristiques, un panorama actuel de l'offre met en évidence une hétérogénéité importante dont l'étude permet de mieux cerner quelques spécificités du géotourisme.
\end{abstract}

Mots-clés: Géotourisme alpin, géosite touristique, médiation scientifique, géosciences.

À la croisée de l'écotourisme et du tourisme culturel, le géotourisme est une niche touristique en pleine expansion. Il s'appuie sur une ressource territoriale, le patrimoine géologique et géomorphologique, et participe à la diversification de l'offre touristique. Son histoire est déjà ancienne puisque H.-B. de Saussure (1740-1799), naturaliste suisse, publiait en 1796 un premier Agenda du géologue-voyageur suivi en 1835 et 1836 des deux tomes du Guide du géologue voyageur d'Ami Boué (1794-1881), géologue français, où l'on pouvait lire dans son tome $1(1835: 1)$ : «Aucune époque n’a été plus fertile en voyages que la nôtre, chaque jour voit s'étendre nos connaissances en géographie géognostique et, en comparant le mode actuel d'observation à ce qui avait lieu au commencement de ce siècle, on s'aperçoit que la géologie a subi une révolution semblable à celle de la civilisation.»

Dès le début du XIX ${ }^{\mathrm{e}}$ siècle, époque de ces premiers apprentis géologues voyageurs, dans certaines régions où la géologie est particulièrement prégnante dans l'environnement, une offre géotouristique voit progressivement le jour. Ainsi, dans le massif alpin, la grotte d'Oliero, au nord de la Vénétie, est la première grotte aménagée pour l'accueil du public dès 1832. Les aménagements, minimalistes, donnent à découvrir, mais pas encore à comprendre. Ils introduisent cependant la mise en scène de ce géopatrimoine souterrain.
Le premier musée de site, le jardin des glaciers à Lucerne, en Suisse, accueille ses premiers visiteurs en 1874. Peu de temps après, en 1892, la gorge du pont du Diable, en Haute-Savoie, s'ouvre à la visite comme les mines de sel de Hallstatt en Autriche au cours de la même décennie. Enfin, plus récemment, le premier sentier d'interprétation géologique, le sentier de Dos Capèl, dans le Trentin, est créé en 1970. Depuis ces premières réalisations, l'offre s'est étoffée et un touriste intéressé par la découverte d'éléments clés de l'histoire géologique de la formation d'une chaîne de montagne n'aura que l'embarras du choix s'il parcourt les Alpes. L'offre se compose, en effet, de quelque 400 sites géotouristiques qui proposent la découverte de géosites naturels ou d'origine anthropique tels que des gisements, affleurements, grottes, gorges, mines ou carrières, mais également des patrimoines géologiques extraits du milieu et qui peuvent désormais être admirés dans des collections au cœur de muséums, de centres d'interprétation ou de collections privées...

Ces sites géotouristiques diffèrent par leurs trajectoires touristiques, les aspects de la géologie qu'ils mettent en valeur ou bien la nature de leur mise en tourisme. Pour le touriste amateur de géologie, ils s'ordonnent, à une échelle plus vaste, en tant qu'éléments d'une histoire géologique, celle de la formation d'une chaîne de montagne. 
Afin de mieux cerner la nature du géotourisme, cet article interroge, dans un premier temps, les logiques d'acteurs qui ont prévalu dans les processus de patrimonialisation puis de mise en tourisme des objets ou des sites géologiques. Pour cela, il s'appuie sur un inventaire de l'offre géotouristique réalisé récemment dans le cadre d'un travail de doctorat. Il questionne, dans un second temps, au-delà de la diversité de l'offre proposée, l'évolution de celle-ci en analysant de l'échelle locale à celle de l'ensemble de la chaîne des Alpes les formes d'élargissement de cette sphère patrimoniale afin de rendre compte de quelques spécificités des pratiques touristiques mises en place.

\section{Diversité des trajectoires de mise en tourisme des géopatrimoines}

Les logiques d'acteurs qui gouvernent les processus de création de l'offre géotouristique peuvent être séparées en trois catégories. Certains sites géotouristiques émergent progressivement d'une histoire locale intimement liée à l'histoire des géosciences. Avant tout sciences de terrain, celles-ci ont conduit de nombreux géologues à visiter des sites contribuant à les rendre célèbres tout d'abord au sein de leur communauté, puis progressivement auprès d'un public plus large. D'autres résultent de la fin de l'exploitation des ressources minières et minérales des Alpes. À la fermeture des mines et des carrières, et dans le souci conjoint de conserver la mémoire du passé et de redynamiser une économie locale en déclin, la mise en tourisme de ces sites a été choisie afin de leur donner une seconde vie. Enfin, le géotourisme ayant acquis une certaine légitimité auprès des acteurs institutionnels locaux et en particulier de ceux du tourisme, des sites, nouvellement découverts ou d'autres qui ne faisaient pas l'objet d'aménagements particuliers, sont devenus les supports de création d'une offre géotouristique.

\section{Des sites géotouristiques qui plongent leurs racines dans I'histoire d'une science de terrain}

À la fin du XVII ${ }^{e}$ siècle, les naturalistes commencent à parcourir les Alpes. Gohau (2000), s'appuyant sur les travaux de Gordon Davies et Marjorie Nicolson, évoque, comme raison principale de ce nouvel intérêt pour des reliefs qui jusque-là avaient plutôt inspiré la répulsion, une composante religieuse associée au développement du protestantisme. La nature, d'une richesse foisonnante devient digne d'intérêt. La proximité entre les grands centres universitaires tels Genève, Zurich, Vienne ou Milan, et le massif alpin explique aussi sa fréquentation par les naturalistes.

Ces voyages permettent des observations de terrain, la récolte d'échantillons et les rencontres entre pairs. Ils vont progressivement jeter les bases de certains des concepts fondamentaux de la géologie et élever cette discipline au rang des sciences empiriques comme la botanique ou la zoologie. Quelques grands noms, parmi ces naturalistes et géologues alpins ont "inventé» des destinations géotouristiques alpines encore d'actualité, non qu'ils aient été les premiers à les découvrir, mais parce que ce sont les premiers à avoir attiré l'attention de leurs contemporains sur celles-ci.

Certains sites géotouristiques actuels ont ainsi une histoire qui remonte à plusieurs siècles. Ils ont d'abord été étudiés par des scientifiques, puis visités lors de congrès internationaux ou de réunions de sociétés savantes. Enfin, leur notoriété s'affirmant, ils ont été valorisés pour permettre leur découverte par un public plus large.

Le Monte Purga di Bolca est situé à environ $25 \mathrm{~km}$ au nord-est de Vérone (voir illustration 1). Il abrite un gisement fossilifère exceptionnel daté d'il y a quelque 46 millions d'années, c'est-à-dire synchrone de la limite Eocène inférieurEocène moyen. Léonard de Vinci (1452-1519) est l'un des premiers à décrire les poissons pétrifiés découverts dans ce gisement. Quelques années après, Gerolamo Fracastoro (1478-1553) attribue les "pierres merveilleuses» à des restes d'anciens organismes marins (Vaccari, 2003). Dès le milieu du $\mathrm{XVI}^{\mathrm{e}}$ siècle, les plus belles pièces font la renommée des cabinets de curiosités en pleine expansion à travers toute l'Europe. Des fossiles du Monte Bolca figurent déjà dans les collections d'Ulyssus Aldrovandi (1522-1605) ainsi que celles de Francesco Calzolari (1522-1609) (Gaudant, 1997). JeanFrançois Séguier (1703-1784), naturaliste nîmois qui séjourne à Vérone sous la protection du marquis Scipione Maffei, de 1736 à la mort de ce dernier en 1755, a tout le loisir d'étudier ces fossiles dont le gisement a été acheté par son hôte. Il en rapportera des exemplaires pour les collections du Muséum d'histoire naturelle de Nîmes (Chapron, 2008). Parallèlement aux investigations scientifiques qui vont permettre de comprendre l'origine du gisement, une activité commerciale d'extraction d'échantillons voit le jour. En 1817, la famille Cerato, arrivée à Bolca dans la seconde moitié du XVIII ${ }^{e}$ siècle, obtient du marquis Antonio Maffei la location et le droit d'exploiter le site désormais appelé «Pesciara » pour en extraire les fossiles. La famille achètera ensuite la carrière et «inventera» le métier de carriers de fossiles. Aujourd'hui, l'offre géotouristique se compose d'un centre d'interprétation situé au cœur du village, créé à l'initiative de la commune de montagne de Lessine et géré par le parc naturel régional du même nom, mais aussi de la visite de la carrière souterraine toujours dirigée par la famille Cerato et qui accueille chaque année quelque 40000 visiteurs. La trajectoire touristique de ce site paléontologique exceptionnel a pris le relais de sa vocation scientifique primitive afin d'en assurer la pérennisation tout en y développant une activité économique qui, au fil du temps, a pris plusieurs facettes.

Le «Haut lieu tectonique suisse Sardona», plus connu par les géologues comme le chevauchement des Glaris (voir illustration 1), vient d'obtenir son inscription sur la liste du patrimoine mondial de l'UNESCO au titre du critère viii, c'est-à-dire en tant qu' "exemple éminemment représentatif des grands stades de l'histoire de la Terre». Au contraire de l'exemple précédent, cette reconnaissance durement acquise marque le point de départ d'une réelle appropriation par les autorités locales de la valeur universelle de ce site reconnu jusqu'à présent par la seule sphère des géoscientifiques.

$\mathrm{Au}$ sud du lac des Quatre Cantons, dans la région de Sargans, les paysages sont marqués par la superposition de roches d'une teinte foncée : un conglomérat verrucano d'âge permien (300-250 Ma) sur des flyschs plissés de couleur claire et bien plus jeunes puisqu'ils se sont formés il y a à peine une trentaine de millions d'années. Cette superposition n'est pas 
ILLUSTRATION 1 : Répartition des sites géotouristiques présentés : 1- Monte Purga di Bolca; 2- Haut lieu tectonique suisse Sardona; 3- Mine de Schwaz; 4- Storie di pietra; 5 - Grotte de la Balme à Collomb et Musée de l'Ours des Cavernes; 6- Sentier du glacier de Morteratsch (carte, et photos 2 à 6 : Nathalie Cayla; photo 1 : Wikipédia).

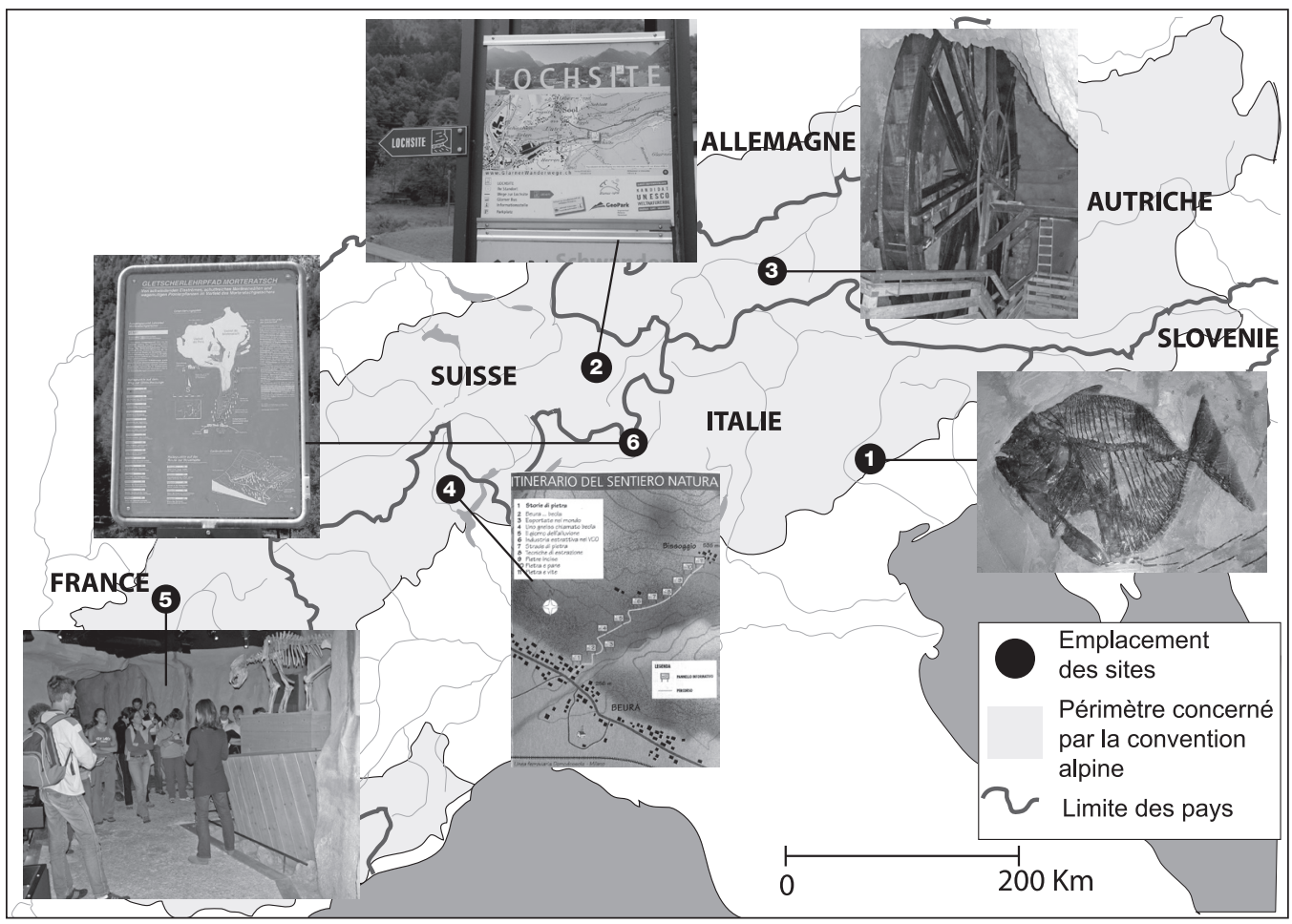

stratigraphique, mais résulte d'un chevauchement devenu célèbre, celui des Glaris.

Hans Conrad Escher Van der Linth, scientifique et homme politique suisse, décrivit pour la première fois cette bizarrerie de la nature en 1812, et en réalisa une aquarelle. Son fils, Arnold (1807-1872), géologue, tente une première explication en 1841, imaginant que le contact anormal observé dans les Glaris est le résultat d'un double pli dont on observe la surface dégagée par l'érosion. Cette interprétation est remise en cause lors du sixième congrès géologique international qui se tient à Zurich en 1894. Deux thèses s'affrontent. Albert Heim (1849-1937), le célèbre géologue suisse, défend celle énoncée par son maître Arnold Escher (Franks et Trümphy, 2005). Il s'oppose en cela aux thèses de Marcel Bertrand défendues, lors de l'excursion de terrain, par Maurice Lugeon (1870-1953) qui affirme que le contact est dû au chevauchement de la couverture du massif de l'Aar glissée vers le Nord à la suite de la compression alpine. Progressivement, le concept des nappes de charriage s'imposera, enterrant définitivement l'immobilisme de la théorie autochtoniste (Fortey, 2004).

Ces débats de spécialistes ont révélé l'histoire d'un paysage qui, au-delà de son étonnante singularité, est l'expression de l'affrontement de forces tectoniques à l'origine de la chaîne des Alpes. Le géoparc suisse Sarganserland Walensee Glarner land, au sein duquel se trouve une grande partie du front de chevauchement, a vu le jour en 1999 dans le cadre d'un projet Regio-plus ayant pour objectif de favoriser le développement régional durable en construisant une offre estivale capable de compléter l'offre touristique locale essentiellement tournée vers le ski. Suite à cette première reconnaissance par un label fédéral et dans le but de renforcer auprès des acteurs locaux la perception du caractère exceptionnel de ce géotope, une demande d'inscription au patrimoine mondial a été déposée en 2004 par le Conseil fédéral. Dans un premier temps celle-ci fut refusée par le comité du patrimoine mondial pour le motif que «la présence d'un chevauchement géologique est une base trop étroite pour l'identification de valeurs universelles exceptionnelles qui caractérisent un bien naturel du patrimoine mondial» (UNESCO, 2005). La candidature a finalement abouti en 2008 dans le cadre d'un dossier renforcé quant au rôle joué par le chevauchement des Glaris dans l'épistémologie des géosciences pour la compréhension d'un concept clé, celui des nappes de charriage.

Le patrimoine géologique, ressource territoriale sur laquelle se construit le géotourisme, relève d'une nature parfois remarquable (Mer de Glace, Cervin, Pénitents des Mées...), mais le plus souvent banale aux yeux du touriste néophyte en géosciences. Seul le travail des spécialistes révèle l'intérêt et donc la richesse d'un affleurement, d'un gisement ou bien d'un paysage. Dans les exemples évoqués ci-dessus, l'intérêt de ces géosites est tout autant lié à leur importance en tant qu'éléments de compréhension de l'histoire géologique d'une chaîne de montagne qu'en tant que lieu clé de l'épistémologie des géosciences. Les jeux d'acteurs ayant concouru à leur patrimonialisation et à leur mise en tourisme s'appuient pourtant sur des logiques différentes. Dans le cas du site fossilifère du Monte Bolca, c'est la nécessité de canaliser un public nombreux en raison de la notoriété du gisement et de prévenir des prélèvements sauvages. Dans celui du chevauchement des Glaris, c'est davantage la nécessité de démontrer aux yeux des acteurs locaux l'intérêt exceptionnel du géotope. 


\section{Des sites géotouristiques qui participent à la reconversion et la diversification de l'offre de certains territoires}

Le sous-sol des chaînes de montagne renferme de nombreuses ressources minières qui, exploitées depuis longtemps, ont très souvent fait la richesse de certaines vallées alpines. Ainsi, les mines de sel de Hallstatt ont donné son nom à la civilisation qui marque le début de l'âge du fer. L'épuisement des gisements, exploités parfois depuis des millénaires, l'opposition croissante des populations locales devant les impacts environnementaux de ces exploitations minières ainsi que la concurrence de pays exploitant des gisements de façon industrielle à l'aide d'une main-d'œuvre bon marché ont progressivement sonné le glas de la plupart des mines et carrières alpines. Le choix de leur mise en tourisme a alors répondu à l'émergence de plusieurs besoins : celui du devoir de mémoire et de patrimonialisation qui correspondait au développement des musées de sociétés (Rasse, 1999), mais aussi la nécessité de maintenir la population dans ces vallées en suscitant l'émergence d'une nouvelle activité économique qui ne renierait pas celles du passé (Deshaies, 2007).

Dans la vallée de l'Inn, au cour du Tyrol et à proximité de la capitale régionale, Innsbruck, les traces de l'activité minière sont encore bien visibles dans le paysage, mais aussi dans l'urbanisme cossu de la petite ville de Schwaz (voir illustration 1). Cette cité a longtemps été un centre économique majeur de l'Empire des Habsbourg en raison de la présence des mines d'argent. À l'apogée de son activité, au début du XVI ${ }^{\mathrm{e}}$ siècle, la mine de Schwaz produisait $85 \%$ de l'argent mondial, assurant la fortune de Jacob Fugger (1459-1525), leur exploitant. Elle employait alors plus de 9000 ouvriers. L'activité extractive a conduit au percement de plus de $500 \mathrm{~km}$ de galeries au cour du massif du Falkenstein. Le minerai imprègne la Schwaz dolomie, à l'origine une vaste plate-forme carbonatée d'âge dévonien appartenant à la zone des grauwackes, socle de la série des alpes calcaires du Nord.

De nombreuses innovations techniques ont vu le jour à Schwaz, notamment dans le domaine hydraulique, avec la mise au point d'un système d'exhaure de l'eau d'infiltration par des conduites et d'une roue hydraulique, indispensable en particulier dans les galeries creusées sous le niveau de la rivière. L'activité minière, progressivement concurrencée par des mines étrangères, a décliné et ne se maintenait, à la fin du $\mathrm{XX}^{\mathrm{e}}$ siècle, que par l'exploitation de la dolomie, ingrédient de la fabrication des revêtements d'asphalte. Une société privée, la Schwazer Silberbergwerk, fondée par des habitants de la ville de Schwaz soucieux de ne pas voir perdre le lien entre la ville et son histoire minière, mais également de créer une activité économique pourvoyeuse d'emplois, développa le projet de création de la mine touristique qui s'ouvrit au public en 1990. Un parcours de $800 \mathrm{~m}$ en train touristique conduit à l'itinéraire pédestre. Celui-ci donne à découvrir les différents postes de travail des mineurs. Le choix a été fait d'un itinéraire ludique au cours duquel le visiteur est pris à parti par un "mineur» exaspéré de ces incessants passages, arrosé par le percement d'une poche d'eau consécutive à un tir de mine et plongé dans une profonde fumée liée à ce même tir. La récente reconstitution grandeur nature de la roue hydraulique installée dans la mine en 1553 est l'un des intérêts techniques majeurs de ce parcours qui attire chaque année plus de 100000 visiteurs (Palme et Ingenhaeff, 1993).

Il existe environ 25 mines touristiques ouvertes au public dans tout le pays, mais seules les mines de sel de Hallstatt, inscrites au patrimoine mondial de l'UNESCO depuis 1997, atteignent une telle fréquentation. Il faut dire que les paysages culturels de Hallstatt-Dachstein/Salzkammergut, outre l'exploitation minière et les témoignages culturels qui y sont associés, dont un saumoduc de quelque $40 \mathrm{~km}$ construit il y a plus de quatre siècles, abritent également un massif karstique qui donne à découvrir trois grottes d'un intérêt majeur : la grotte de Köppenbrüller, celle du Mammouth et enfin la grotte glacée de Rieseneisehöhle. Cette offre est complétée par un sentier d'interprétation géologique qui conduit au point de vue des cinq doigts, plate-forme d'observation plongeant littéralement le visiteur au cour de ce paysage grandiose. On peut dans ce cas parler d'un véritable géoterritoire touristique : à la découverte d'un grand massif calcaire s'ajoute celle de la mine qui a fait la richesse de la région et d'un site archéologique renommé. Ces différents éléments gérés de façon indépendante sont désormais associés dans une offre touristique globale améliorant la lisibilité géotouristique de ce territoire.

Le nord-ouest du lac Majeur est une région du Piémont bien connue des géologues en raison d'un affleurement de croûte continentale mettant au jour les zones les plus profondes de celle-ci (voir illustration 1). C'est aussi un lieu important d'extraction de pierres ornementales. À l'origine exploité pour le marché local, ce secteur économique s'est considérablement développé après la Seconde Guerre mondiale grâce à l'arrivée du chemin de fer. Les principales roches extraites des vallées de l'Ossola sont : les granites tardi-hercyniens de Baveno (dénommé Rose), de Montorfano (Blanc) et de Mergozzo (Vert) extraits des plutons de Mottarone et du Montorfano (Alciati, 2008b); les marbres paléozoïques de Candoglia et d'Ornavasso; les Beole et Serizzi, orthogneiss qui résultent du métamorphisme alpin de granites varisques (Alciati, 2008a) enfin les Creole, marbres dolomitiques mésozoïques de la zone pennique (Alciati, 2008c). 69 carrières sont encore en activité; elles emploient en moyenne chacune 5 à 6 personnes. Le bassin de Verbano Cusio Ossola représente $60 \%$ de la production du Piémont en pierres ornementales (Filipello, 2004).

L'importance économique de cette activité extractive ainsi que l'intérêt patrimonial de ces pierres ornementales qui caractérisent le bâti de la région frontalière du Val d'Ossola et du sud du Tessin a donné naissance, entre 2000 et 2006, au programme européen «Interreg IIIA : Osservatorio Sub-Alpino Materiali Territorio Restauro (OSMATER)» entre la Suisse et l'Italie. Plusieurs objectifs y étaient attachés. Tout d'abord, le développement de l'activité économique passait par une meilleure structuration de la filière d'exploitation des pierres ornementales. Dans une perspective de valorisation patrimoniale, un second objectif était de créer une banque de données sur l'architecture et l'utilisation des pierres, mais aussi de réfléchir à la mise en place d'une offre géotouristique liée à ce patrimoine géologique et historique. Différents partenaires scientifiques, dont le département des sciences de la Terre de l'Université de Turin, se sont investis dans cette partie du projet. Après la phase 
d'inventaire et d'analyse, six itinéraires géotouristiques ont été proposés, et un écomusée a ouvert ses portes. Non loin de là, un sentier dénommé "Storie di Pietra» a aussi été développé en partenariat avec le parc national du Val Grande. Ce vaste programme implique des acteurs du monde économique (carriers, professionnels du tourisme), des scientifiques (laboratoires de mécanique des roches, mais également spécialistes des sciences de la Terre) ainsi que des acteurs locaux. Ce partenariat original assure un ancrage territorial qui permet le développement de pratiques géotouristiques sur les lieux d'une activité d'extraction encore active. Cette complémentarité permet non seulement de mieux faire comprendre l'intérêt économique de la filière de production des roches ornementales, mais assure une diversification de l'offre touristique, gage d'une durabilité plus grande de cette offre.

Une enquête conduite en 2007 dans le cadre du projet «Iron Route» auprès de 54 mines touristiques réparties dans 10 pays européens révèle que la majorité des exploitations ont cessé leur activité au cours des années 1980. Les projets de reconversion ont alors accompagné cette cessation d'activité, principalement à l'initiative des autorités locales et donc soutenues par des financements publics (Busolini, 2007). Alors que certains de ces gisements sont particulièrement intéressants en raison des conditions de leur mise en place représentatives de différents épisodes de l'orogenèse alpine, force est de constater que cet aspect géologique reste souvent secondaire dans le discours patrimonial élaboré pour la mise en tourisme. Ce dernier s'appuie souvent davantage sur un registre culturel mettant en avant l'histoire humaine liée à ces exploitations. Cet aspect parcellaire de la médiation ainsi réalisée s'explique en partie par l'origine des acteurs institutionnels chargés du patrimoine. La nécessité d'une approche pluridisciplinaire associant géoscientifiques, historiens, ingénieurs, sociologues et didacticiens est un moyen de garantir une mise en valeur qui permettra aux différents visiteurs de multiplier l'intérêt de leur découverte. En effet, les mines et carrières sont des patrimoines aux multiples facettes qui révèlent à la fois des éléments de l'histoire géologique, de l'histoire des techniques, de l'histoire de l'art, mais également de l'histoire des hommes à l'échelle locale et globale, l'économie des matières premières étant largement dépendante des échanges à l'échelle planétaire depuis de nombreux siècles.

\section{La capacité du géotourisme à investir de nouvelles découvertes}

Au-delà des sites historiques qui viennent d'être évoqués, les recherches permanentes qui sont conduites au cœur du massif alpin révèlent régulièrement de nouveaux sites importants pour la compréhension de l'histoire de cette chaîne de montagne. Ce sont parfois des sites totalement ignorés, mais souvent aussi des sites déjà connus et parcourus depuis longtemps sans pour autant qu'une attention particulière ait été portée à leur dimension géologique. La montée en puissance de la reconnaissance des géopatrimoines ainsi que le développement du géotourisme se traduit par une mobilisation de la part des acteurs, lesquels considèrent ces objets géologiques comme des ressources pouvant donner lieu au développement de pratiques géotouristiques capables de différencier leur territoire.
La grotte de la Balme à Collomb, dans le parc naturel régional de Chartreuse, est traversée par des milliers de touristes chaque année, car elle se trouve sur le tracé de l'ascension du mont Granier (voir illustration 1). C'est pourtant au fond de celle-ci qu'à l'automne 1988 des membres du spéléo-club de Savoie découvrent l'entrée d'une galerie comblée par un éboulement. Le passage alors dégagé donne accès à un gisement d'ours des cavernes qui s'étend sur une superficie de près de $3000 \mathrm{~m}^{2}$. Les fouilles menées durant six étés consécutifs à partir de 1989 ont été conduites par Michel Philippe, responsable des sciences de la Terre au Muséum d'Histoire naturelle de Lyon. 12000 ossements en ont été extraits, alors que seul $10 \%$ de la surface du gisement a été fouillé. Ils constituent une collection conservée au Muséum de Lyon. Le reste du gisement a été laissé en l'état pour des prospections futures.

La nature des ossements retrouvés (en particulier l'âge des oursons d'après la dentition) permet de préciser que cette grotte était une grotte à hivernation dotée d'une «nurserie». L'extension verticale du gisement indique que la grotte fut occupée environ de 45000 à 24000 ans avant J.-C., soit durant quelques 21000 ans (Philippe et al., 2004). L'éboulement de l'entrée de la grotte, qui n'a pas encore été localisée, a isolé à l'époque ce gisement, le protégeant ainsi de toute perturbation ou destruction.

Au pied du Granier, au cour de la commune d'Entremont-le-Vieux, en Chartreuse, dans le parc naturel régional du même nom, vivent quelque 500 habitants. L'activité touristique y est en déclin en raison d'un manteau neigeux de plus en plus incertain en hiver. La pluriactivité saisonnière y est donc menacée, mettant en péril l'activité agricole souvent conduite par des «double-actifs». Cette découverte paléontologique est rapidement pressentie par les autorités locales, et en particulier par le maire Michel Claret, comme un moyen de redynamiser le tourisme dans la vallée. Un centre d'interprétation, le Musée de l'Ours des Cavernes en Chartreuse, est ouvert en 2002, au cour du village. 1,5 million d'euros sont investis pour le bâtiment et la muséographie est financée à $37 \%$ par des fonds européens (FEDER), 25\% par des fonds nationaux (FNADT), 19\% par la région Rhône-Alpes, 13\% par le Conseil Général de la Savoie et $6 \%$ en autofinancement communal. D'une superficie d'environ $600 \mathrm{~m}^{2}$, ce centre d'interprétation n'expose que des moulages des ossements découverts. L'aménagement intérieur est conçu comme l'intérieur d'une grotte dans le style des expositions d'immersion. Le visiteur est amené à travers divers indices à comprendre le mode de vie de l'ours des cavernes, le climat qui régnait à son époque, la formation du gisement... Le mécénat scientifique d'EDF a financé en 1998 les relevés nécessaires à la reconstitution 3D de la grotte qui est présentée dans un film en relief au cour du dispositif muséographique. Des événementiels assurent le renouvellement de l'offre, créant de l'interactivité avec les visiteurs dont la fréquentation est d'environ 22000 par an. Une coopérative laitière moderne est aussi ouverte à proximité bénéficiant désormais de ce flux de visiteurs qui assure par la vente directe une meilleure rentabilité à la production fromagère locale. 10 années ont donc suffi à cette commune pour développer une offre touristique qui a permis de redynamiser l'ensemble de son activité économique en s'appuyant 
sur une découverte scientifique certes importante, mais qu'il y a encore quelques années aurait pu rester sans conséquences pour la société civile.

Dans d'autres cas, ce n'est pas une découverte qui est à l'origine d'une nouvelle offre, mais tout simplement l'étude plus poussée d'un site géologique ou géomorphologique. Ces investigations remettent à l'honneur un site déjà connu, mais qui n’avait pas été réfléchi en tant que site géotouristique. Elles attirent l'attention des autorités locales et peuvent déboucher sur des projets de développement d'une offre géotouristique.

Le glacier de Morteratsch est le plus grand glacier des Grisons avec une longueur de 6,4 km et un volume de $1,2 \mathrm{~km}^{3}$ en 2008 (voir illustration 1). Il est dominé par le Piz Bernina qui culmine à $4089 \mathrm{~m}$. La première photographie du glacier date de 1867. Il a été ensuite régulièrement suivi depuis 1878 par le comité glaciologique helvétique, le Gletscherkollegium, créé en 1869. Son recul, marqué par cinq pauses, n’a pas cessé depuis cette époque. La comparaison de photos du sentier prises tout au long du XXe siècle illustre ce retrait ainsi que la reconquête des moraines par la végétation modifiant le paysage. Max Maisch, glaciologue de l'Université de Zurich, s'est intéressé à ce glacier dès 1978 et a conçu, peu après, l'idée d'un sentier d'interprétation qui viendrait enrichir le parcours qui, depuis la station ferroviaire de Morteratsch, conduit jusqu'à la langue glaciaire actuelle. Ce projet, soutenu par la commune de Pontresina, a vu le jour en 1993. Il présente une succession de 20 arrêts matérialisés par des panneaux indiquant la date à laquelle le glacier atteignait le point en question. Les informations scientifiques sont diffusées au moyen de deux fascicules : le premier présente simplement les différents points d'arrêt et les observations associées, le second replace les données concernant le glacier dans une perspective plus régionale présentant les travaux scientifiques réalisés par le laboratoire (Maisch et al., 1999). Plus récemment, l'École polytechnique fédérale de Zurich vient de créer une interface web (voir : http://www. klimaweg.ethz.ch/) entièrement dédiée à la compréhension de l'impact du réchauffement global. Celle-ci consiste en une balade virtuelle éducative le long d'un sentier alpin au départ, là encore, de Pontresina.

Les géoscientifiques prospectent sans cesse le terrain, mettant au jour de nouveaux géosites ou bien réinterprétant des sites déjà bien connus. Ces découvertes qui enrichissent régulièrement le patrimoine géologique des territoires sont désormais perçues par les autorités locales comme susceptibles de diversifier également l'offre touristique les conduisant, dans un souci constant de se démarquer des territoires voisins, à initier des projets de création d'une offre géotouristique. Si cette volonté est louable au regard de certaines réussites très médiatisées telle la découverte d'Ötzi, l'homme des glaces, en 1991, à l'origine de deux centres d'interprétation, en Italie et en Autriche, d'un musée à Bolzano et de plusieurs sentiers d'interprétation, elle ne doit pas tendre à multiplier une offre banalisée, mais bien à révéler la représentativité d'un lieu. Les deux exemples présentés témoignent du caractère extensif des ressources géologiques susceptibles d'être patrimonialisées : il existe un lien direct entre l'évolution des sciences et des techniques d'investigation, la connaissance scientifique des sites géologiques et les dynamiques de patrimonialisation. Les investigations scientifiques doivent donc toujours être encouragées : de nouvelles méthodes d'études, de prélèvement ou d'analyse peuvent, en effet, éclairer d'un regard neuf un site déjà connu ou bien être à l'origine de la découverte de nouveaux sites, renouvelant ainsi le patrimoine géologique.

Loin d'être figé dans le temps, le patrimoine géologique est un patrimoine vivant, dans le sens où le développement des géosciences assure en permanence la découverte de nouveaux géotopes ainsi que l'amélioration de la connaissance de ceux déjà inventoriés. Les scientifiques occupent donc un rôle central dans le développement du géotourisme, intervenant à différentes étapes du processus de mise en tourisme. Chaque réalisation suit une trajectoire dépendant de la ressource patrimoniale, du jeu des acteurs aux différentes échelles territoriales, mais également d'une dynamique plus globale qui progressivement a fait reconnaître l'intérêt des géopatrimoines parmi les professionnels du tourisme. L'offre géotouristique qui prend alors forme s'articule autour de quelques invariants : musées, parcours souterrains, sentiers d'interprétation. Au-delà de ces outils et dans le souci de mieux cerner les particularités du géotourisme, la seconde partie de cet article tente d'éclairer certaines singularités de la mise en tourisme du patrimoine géologique et géomorphologique.

\section{Diversité des procédés de mise en géotourisme}

Différents produits géotouristiques composent l'offre des territoires. Certains font découvrir des géosites par le biais d'une offre marchande. Ces géosites peuvent être d'origine naturelle (grottes, gorges ou canyons) ou bien d'origine artificielle comme d'anciennes exploitations, telles des mines ou des carrières. Une offre non directement marchande existe aussi, avec les sentiers d'interprétation géologique. Elle associe à la randonnée une offre culturelle par des panneaux, des fascicules ou des données téléchargeables sur téléphone intelligent. Enfin, si les sites naturels sont inaccessibles ou bien si les patrimoines géologiques en ont été extraits, des écomusées, centres d'interprétations, collections ou muséums peuvent être visités.

À ces produits pérennes sont également associés des événements qui proposent au visiteur une expérience originale de découverte.

Au cours du temps, le géotourisme, d'abord tourné vers une observation contemplative des sites, a progressivement donné toute sa place à la médiation géoscientifique indispensable à la bonne compréhension de ceux-ci.

Au-delà de la diversité de l'offre, certaines singularités peuvent être relevées, elles vont être présentées en développant l'étude de trois exemples : l'analyse de l'offre au travers de critères culturels; l'évolution du tourisme souterrain qui, sur plus de deux siècles d'existence, a considérablement diversifié son offre; enfin, l'étude des sentiers d'interprétation dont le nombre, près de 150 dans l'arc alpin, autorise l'approche comparative.

\section{Deux approches culturelles différentes du géotourisme}

L'analyse de la répartition des 400 sites géotouristiques recensés dans l'arc alpin montre une certaine hétérogénéité, tant dans les formes que dans les thèmes retenus pour la mise en 
tourisme. La répartition observée peut en partie s'expliquer par des différences culturelles relativement marquées entre pays latins et germaniques.

L'Allemagne, l'Autriche ainsi qu'un certain nombre de cantons suisses privilégient les pratiques touristiques qui permettent de découvrir sur le terrain le patrimoine géologique. Ces pays possèdent un réseau important de sentiers d'interprétation sur le thème des géosciences et ont ouvert de nombreuses mines, carrières, affleurements, gorges ou grottes aux pratiques géotouristiques. Le public est d'ailleurs au rendezvous puisque c'est dans ces pays que l'on trouve les fréquentations géotouristiques les plus élevées, 350000 pour la mine de Berchtesgaden, 300000 pour les gorges de Breitachklamm, 100000 pour chacune des grottes du Dachstein... Deux raisons peuvent expliquer cet état de fait : un attrait marqué des habitants pour les pratiques sportives de pleine nature en raison d'une approche naturaliste plus développée dans ces pays, mais aussi l'origine des acteurs mobilisés par ce champ patrimonial. Ainsi, en Autriche, le Club Alpin national a joué un rôle particulièrement important dans le développement des sentiers d'interprétation du pays.

La France, l'Italie, mais aussi un canton comme le Valais, en Suisse, ont investi ce champ par une approche plus culturelle en développant un important réseau de musées abordant le thème des géosciences. Les investissements sont alors souvent plus importants, et la rentabilité du produit sur le long terme doit pouvoir être assurée. Cette approche peut s'expliquer par une sensibilité plus grande à l'aspect culturel associée aux processus de patrimonialisation, mais aussi par la nature des acteurs mobilisés qui sont davantage issus du monde de la culture. L'échelle territoriale décisionnelle est aussi importante : seuls des départements ou des régions peuvent supporter de tels investissements publics, puis assurer la gestion, parfois déficitaire, de tels outils. Là encore le public est cependant souvent au rendez-vous puisque le Muséum d'histoire naturelle de Milan attire 350000 visiteurs par an et le Musée de préhistoire des gorges du Verdon, dans le département des Alpes de Haute-Provence, plus de 70000.

Ainsi, deux types de grandes pratiques géotouristiques apparaissent :

1- l'une, principalement développée dans les pays germanophones, qui s'appuie sur une approche de terrain et qui enracine le géotourisme au cour des pratiques écotouristiques;

2- l'autre, principalement développée dans les pays latins, qui s'appuie sur une approche muséographique, donc plutôt délocalisée, et qui enracine le géotourisme au cœur du tourisme culturel.

Ces approches ne s'excluent pas bien sûr l'une l'autre et sont plutôt à considérer dans la perspective de la construction d'une offre géotouristique qui répondrait aux attentes de la diversité des touristes attendus sur un territoire.

\section{Les visites de grottes : évolution de l'offre du contemplatif à l'explicatif}

Selon Zhang et Jin (1994), il existe actuellement près de 800 grottes aménagées dans le monde qui accueillent environ 170 millions de visiteurs par an et génèrent un chiffre d'affaire d'environ 1,5 milliard de dollars. Près de 100000 personnes travaillent de façon directe à l'exploitation d'une cavité touristique, auxquelles il faut ajouter les nombreux emplois indirects générés (Cigna et Burri, 2000). Ce secteur géotouristique est donc remarquable par son ancienneté qui permet de reconstituer son évolution, par l'abondance de son offre qui autorise l'approche comparative, mais également par l'importance de sa fréquentation montrant qu'il répond bien à l'attente du public.

La mise en tourisme des grottes est un phénomène déjà ancien puisque la première cavité à ouvrir au public dans les Alpes fut la grotte d'Oliero en 1832. Située en Vénétie, elle a été découverte quelques années auparavant par Alberto Parolini (1788-1867) qui, le premier, en traversa le lac souterrain. Ce naturaliste, qui avait étudié à Padoue puis parcouru l'Europe à la rencontre d'autres savants, rapporta de l'une de ses expéditions à la grotte de Postojna (Slovénie) des protées qu'il relâcha dans celle d'Oliero, multipliant ainsi les sources d'intérêt à la visite de la cavité. La renommée est rapide et, au printemps 1834, George Sand (1804-1876) visite le site. Elle décrit ainsi son expérience :

Trois grottes d'une merveilleuse beauté pour la forme et les couleurs du roc occupent les enfoncements de la gorge. L'une a servi longtemps de caverne à une bande d'assassins; l'autre recèle un petit lac ténébreux que l'on peut parcourir en bateau et sur lequel pendent de très belles stalactites. Mais c'est une des curiosités qui ont le tort d'entretenir l'inutile et insupportable profession de touriste. Il me semble déjà voir arriver, malgré la neige qui couvre les Alpes, ces insipides monotones figures que chaque été ramène [...] véritable plaie de notre génération qui a juré de dénaturer par sa présence la physionomie de toutes les contrées du globe, et d'empoisonner toutes les jouissances des promeneurs contemplatifs, par leur oisive inquiétude et leurs sottes questions (p. 39)

Comme elle le raconte ici, il semble qu'à l'époque ce soit davantage l'expérimentation du monde des ténèbres qui attire les visiteurs que la recherche d'une quelconque compréhension de celui-ci, mais il faut dire que la karstologie n'en est alors elle-même qu'à ses balbutiements. Depuis, les choses ont changé puisque la commune, en partenariat avec le groupe spéléologique local a ouvert, en plus de la visite des grottes en 1994, un musée du karst qui propose de mieux faire comprendre la circulation des eaux souterraines, la nécessité de leur protection et les contraintes d'aménagement que cela suppose. Aujourd'hui, plus de 30000 visiteurs viennent chaque année à la découverte de ce site.

Cette première vague de mise en tourisme restera limitée, puisqu'à la fin du XIX ${ }^{e}$ siècle seules neuf grottes sont ouvertes au public (voir illustration 2). Parmi celles-ci, certaines présentent déjà des aménagements modernes. Ainsi la grotte de Kraus, en Autriche, bénéficie d'un éclairage artificiel dès 1884. Il faut dire que quelques sites majeurs, en dehors de l'arc alpin, telle la grotte de Postojna, en Slovénie, ouverte au public dès 1819 , drainent à l'époque des flux de touristes importants alors que le tourisme alpin n'en est qu'à ses balbutiements (Duval, 2007). 


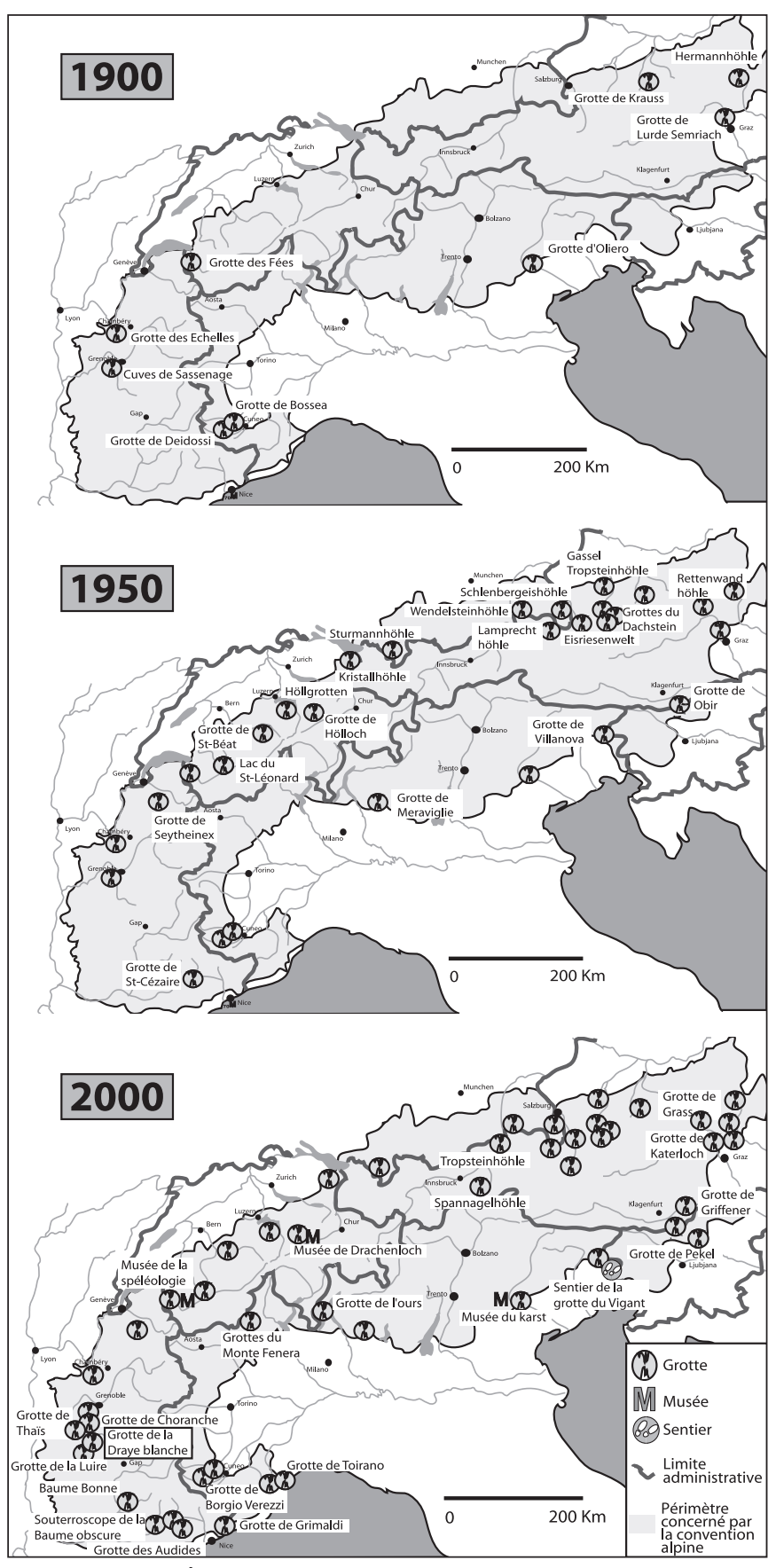

ILLUSTRATION 2 : Évolution du nombre de grottes touristiques dans l'arc alpin (source : compilation de l'auteure).

Au début du siècle dernier, on observe une rapide évolution de ce panorama. Le nombre de cavités découvertes progresse avec le développement des explorations souterraines, et la mise en tourisme est parfois envisagée en raison de la réussite des premières réalisations (Gauchon, 1997). Toutefois, à cette époque, l'activité touristique n'a souvent qu'un objectif lucratif sans considération quant à la vulnérabilité de la ressource sur laquelle elle s'est construite. Certains aménagements sont ainsi à l'origine de dégradations irréversibles des sites. Celles-ci sont parfois intentionnelles : taille de marches d'escalier dans des planchers stalagmitiques, mais elles sont le plus souvent liées à une méconnaissance de la vulnérabilité de l'environnement karstique; perturbation des paramètres physicochimiques et thermiques souterrains causée par la présence humaine ou bien par des systèmes d'éclairage induisant la prolifération de micro-organismes ou des modifications dans les modalités du concrétionnement.

Dès la première moitié du XXe siècle, une vingtaine de grottes touristiques complètent l'offre déjà présente dans l'arc alpin, toutes situées près de centres touristiques importants ou bien près d'axes de circulation majeurs (voir illustration 2).

Jusque dans les années 1990, ce nombre va progresser, témoignant de l'engouement du public pour ce type d'offre, et atteindre la valeur actuelle de 51 dans l'ensemble de l'arc alpin. La dernière grotte à avoir ouvert son réseau souterrain, en 1991, est celle d'Obir, en Carinthie. Son originalité tient au fait que le réseau karstique a été découvert par des mineurs qui exploitaient des filons de fer et de plomb du massif. Un temps ouvert à la visite pour les curistes qui prenaient les eaux à Bad Eisenkapel, juste après la fermeture de l'exploitation minière en 1902, le réseau fut redécouvert en 1985, et la visite aménagée attire depuis plus de 40000 visiteurs par an.

L'offre actuelle est extrêmement hétérogène. Quoi de commun entre la grotte de Kraus dans le géoparc Eisenwurzen ou la grotte de Hölloch dans le canton de Schwyz qui accueillent chacune quelques milliers de visiteurs par année munis de casques et d'un éclairage individuel (car ces grottes n'ont plus d'aménagement électrique pérenne), et la grotte du Mammouth dans le massif du Dachstein ou celle de Choranche dans le Vercors (Biot, 2006) qui attirent chacune plus de 100000 visiteurs autour d'un nouveau son et lumière utilisant les techniques de spectacle laser les plus innovantes afin de renouveler leur produit touristique?

Là encore, loin des produits standardisés propres à certaines destinations touristiques, le géotourisme souterrain offre de nombreuses possibilités (Hobléa, 2008). Ces dernières vont de la visite contemplative où l'intérêt est avant tout soutenu par des techniques de mise en scène multimédias qui relèvent du spectacle à la pratique de la spéléologie avec des guides confirmés où les apprentissages techniques se conjuguent avec la découverte de quelques éléments fondamentaux du milieu karstique.

L'ancienneté du tourisme souterrain permet de retracer les grandes étapes de son évolution et ainsi de mieux comprendre les motivations qui ont conduit à la mise en tourisme de ces espaces. Jusqu'au début du XX $\mathrm{XX}^{\mathrm{e}}$ siècle, les grottes sont aménagées en raison de leur proximité avec un axe de circulation. C'est le cas de la grotte des Échelles en Savoie qui, avant le percement du tunnel du même nom, était située au bord de la route. Les grottes peuvent aussi être liées à un lieu de villégiature, telle la grotte d'Oliero à proximité de Venise. Au début du XXe siècle, l'offre s'enrichit et les aménagements sont réalisés dans des sites qui possèdent des éléments remarquables. Ainsi Eisriesenwelt, une grotte glacée du massif du Tennengebirge, dans la région de Salzbourg, est ouverte aux visiteurs en 1920. Une route d'accès est aménagée en 1954 et un téléphérique est construit en 1955. La présence de la glace à l'intérieur de la cavité en est un 
ILLUSTRATION 3 : Analyse

de l'offre de sentiers géodidactiques dans l'arc alpin (source : compilation de l'auteure).

\begin{tabular}{|l|c|c|}
\cline { 2 - 3 } & $\begin{array}{l}\text { Part de la surface } \\
\text { nationale concernée par } \\
\text { la Convention alpine (\%) }\end{array}$ & $\begin{array}{l}\text { Part de l'offre de sentiers } \\
\text { géologiques (\%) }\end{array}$ \\
\hline Allemagne & 6 & 8,6 \\
\hline Autriche & 28,5 & 23 \\
\hline France & 21,5 & 20,7 \\
\hline Italie & 27,5 & 25 \\
\hline Slovénie & 3,5 & 2 \\
\hline Suisse & 13 & 20,7 \\
\hline
\end{tabular}

Répartition par pays

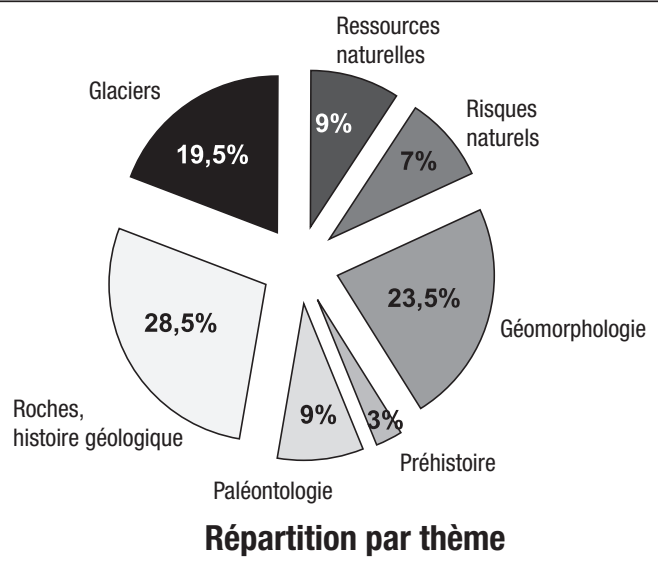

Répartition par thème élément remarquable. Le tourisme se développe alors dans les Alpes, il faut offrir de quoi occuper les excursionnistes et les aménagements même coûteux s'avèrent désormais rentables. Pas toujours d'ailleurs, comme en témoigne la fermeture de la grotte d'Obir dont il a été question précédemment. Le maillage du territoire alpin progresse et, depuis quelques années, l'évolution du tourisme souterrain n'est plus quantitative, mais s'est déplacée vers la création de produits touristiques plus complets. C'est le cas de la visite de la grotte de Villanova, dans le Frioul, à laquelle est associée la découverte d'un sentier karstique ou bien la diversification du produit sous la forme de différents types de visites. Dans cette phase de diversification, on peut noter les efforts réalisés par certains gestionnaires pour donner une place plus importante aux géosciences aux côtés de celle longtemps assignée à la fantasmagorie du monde souterrain. Ainsi, les guides souvent choisis parce que polyglottes bénéficient désormais, comme aux grottes de Choranche, d'une formation de base en karstologie. Ces nouvelles formes de pratiques touristiques font évoluer le géotourisme en renforçant les aspects éducatifs qui lui sont associés. On observe aussi une segmentation de l'offre développant des visites adaptées aux attentes des différents publics et de leur niveau de connaissance et de pratiques du monde souterrain.

\section{Les sentiers d'interprétation : des outils d'apprentissage des géosciences sur le terrain}

La randonnée occupe une place de choix dans l'ensemble des activités réalisées par des touristes en villégiature dans les Alpes et, face à cette demande, toutes les destinations touristiques se sont équipées d'itinéraires, de la simple promenade à la randonnée sportive. Le développement des sentiers thématiques, de découvertes, de didactiques ou encore de parcours d'interprétation, au gré des évolutions de la nomenclature, a permis à partir des années 1970, souvent sur le territoire d'espaces naturels protégés, de conjuguer les objectifs d'information et d'éducation à l'environnement avec l'essor des pratiques sportives de pleine nature. Le développement récent de cette nouvelle offre montre la progression des pratiques géotouristiques vers des formes d'apprentissage sur le terrain. Non directement marchande, cette offre est un moyen de mettre en valeur certains territoires à partir de ressources qui ne sont pas exceptionnelles, mais qui méritent d'être rendues intelligibles par le biais de l'interprétation.

L'origine de ces sentiers à thème est ancienne puisque, comme le rappelle Thomas Hose dans un récent article (Hose, 2008) le premier geotrail, sentier d'interprétation de la géologie, a vu le jour, en Angleterre en 1881, dans la ville de Rochdale (Lancashire). En 1925, les États-Unis en ont créé un dans le parc national Palisade Intestate et, en 1930, l'Allemagne, dans la forêt de Bredower dans le Land de Brandebourg. À partir des années 1960, ces créations se généralisent. Ainsi, l'Allemagne possède 1080 sentiers à thème dont la majeure partie se situe en Bavière. Les principaux thèmes abordés sont : la forêt (35\%), les biotopes $(28 \%)$, les paysages $(18 \%)$, la géologie et la géomorphologie $(7 \%)$, l'histoire $(5,5 \%)$ et des thèmes multiples pour les 6,5\% restant (Kestler, 2005). En Autriche, il en existe plus de 700. Parmi ceux-ci, $9 \%$ portent sur la géologie (Eder, 2007). Dans ce pays, un portail internet a été créé par le ministère de l'Environnement donnant accès par région et par thème à une base de données de 350 de ces sentiers (voir : http://lehrpfade. lebenministerium.at). Quant à la Suisse, Suisse Tourisme recensait 300 sentiers à thème en 1999 (Rohn-Brossard, 2006). Au cour de cette offre, les sentiers abordant les géosciences sont minoritaires. Malgré tout, cette offre est conséquente puisque dans les Alpes, 140 sentiers géodidactiques ont été recensés. Leur répartition géographique (voir illustration 3) montre que la Suisse, avec près de $21 \%$ de l'offre sur seulement $13 \%$ de la superficie comprise dans la convention alpine, est un pays où la pratique de «l'itinérance éducative» au service des géosciences est particulièrement développée. Quant aux thèmes repris par ces sentiers, on constate que la géomorphologie, en y incluant les thèmes des risques naturels et des glaciers, est largement majoritaire au cœur de cette offre. Cela s'explique par la prédominance de l'approche paysagère dans la plupart des dispositifs d'interprétation, mais également par l'implication et la forte mobilisation de la communauté des géomorphologues dans l'aventure de la diffusion du savoir et de la mise en valeur des territoires.

Différentes signalétiques sont utilisées : des panneaux interprétatifs qui parfois atteignent des tailles incompatibles avec le respect du paysage et des balises chiffrées qui nécessitent d'être en possession d'un fascicule d'interprétation. Ces différents 
supports sont diversement appréciés par les visiteurs. Dans son étude sur l'évaluation de l'offre dérivée auprès de publics cibles, Jean-Pierre Pralong montre que dans le cas des gorges de la Diosaz, en Haute-Savoie, $40 \%$ des visiteurs plaident en faveur de panneaux didactiques, $27 \%$ pour des livres ou brochures et $15 \%$ pour des visites guidées (Pralong, 2006). Le renouvellement de cette offre de sentiers semble osciller désormais entre deux extrêmes : la disparition de la signalétique de terrain remplacée par des guides GPS-touristiques numériques; des flash-codes apposés sur des bornes; ou bien, à l'opposé, la création d'un véritable mobilier de sentier permettant de développer l'offre d'interprétation en rendant le randonneur actif dans une découverte plus ludique. La diversification actuelle de l'offre de sentier porte donc sur la nature et la qualité de la médiation scientifique développée.

L'apparition, relativement récente dans l'offre géotouristique de l'arc alpin, des sentiers d'interprétation a été suivie d'une inflation de cette offre associée à une certaine banalisation. Différents acteurs sont à l'origine de ces projets : des acteurs des espaces naturels protégés qui ont pour objectif l'éducation à l'environnement, des scientifiques qui souhaitent valoriser leurs travaux auprès du plus grand nombre ou bien des collectivités territoriales qui voudraient diversifier leur offre touristique. Rares sont les productions réalisées en synergie, d'où parfois des résultats décevants au regard des fonds investis. En effet, la qualité d'une réalisation dépend de plusieurs facteurs dont la qualité et la spécificité des processus de médiation qui doivent être en adéquation avec les projets territoriaux des acteurs locaux. Ainsi, si l'on analyse les outils de médiation développés dans le cas des 30 sentiers d'interprétation portant sur le thème des glaciers, trois tendances peuvent être observées : des sentiers militants avec pour objectif la prise de conscience par le randonneur de l'impact du changement global sur les glaciers alpins; les sentiers éducatifs dans lesquels l'accent est mis sur la transmission d'un savoir de base permettant au randonneur d'interpréter les paysages glaciaires; enfin des sentiers que l'on pourrait qualifier de patrimoniaux, qui mettent en valeur spécifiquement un site naturel en relation avec son histoire propre, celle de sa vallée et des habitants qui y ont vécu. Les techniques didactiques utilisées lors de la création des différents supports de médiation s'appuient, plus ou moins efficacement, sur différents ressorts de la diffusion des savoirs : reformulation du discours scientifique, types de transposition, raisonnements inductifs ou déductifs, analyse scrypto-visuelle... (Jacobi, 1999). Peu de travaux scientifiques ont porté sur l'évaluation de ces différents produits (Berrebi, 2006) ou bien sur les processus de leur création (Sellier, 2009). Il semble désormais nécessaire d'améliorer la qualité de ces outils afin de mieux répondre aux attentes du public.

Les pratiques touristiques associées au développement du géotourisme empruntent au tourisme culturel et à l'écotourisme des outils de découverte qui sont ensuite adaptés au contexte géologique à mettre en valeur. Ce n'est cependant pas ce contexte qui détermine le type d'outil de médiation choisi, mais bien davantage le contexte culturel au sein duquel cette réalisation est élaborée.

Le tourisme souterrain, lié à la découverte du karst, permet de suivre l'évolution de l'offre depuis plus de deux siècles. À une période contemplative liée à quelques sites spectaculaires et bien situés a fait suite une phase de diversification mettant en valeur, grâce aux travaux des karstologues et des spéléologues, des réseaux plus retirés et pour certains plus banals. Les outils de médiation ont aussi connu une réelle évolution mettant en avant des aspects spectaculaires, aventureux et plus récemment éducatifs. Ainsi, un même site peut désormais répondre aux différentes attentes du public avec des parcours diversifiés.

Le développement des sentiers d'interprétation géologique dont un panel de quelque 150 réalisations qui a été recensé dans les Alpes autorise l'analyse comparative. Celle-ci met en évidence un foisonnement des pratiques qui nécessiterait peut-être désormais une réelle réflexion quant à la qualité des productions afin de mieux faire connaître les «bonnes pratiques» et d'améliorer ainsi les projets à venir.

\section{Conclusion}

La reconnaissance de la valeur patrimoniale des objets géologiques et géomorphologiques a permis de les faire accéder au statut de ressource patrimoniale. Ils sont devenus, parfois depuis longtemps, les supports du développement de pratiques touristiques. Les logiques d'acteurs à l'origine de cette offre sont extrêmement variées : origine des initiatives (publics ou privées), échelle de réalisation (locale, régionale ou nationale), objectif visé (économique, culturel, patrimonial...). $\mathrm{Au}$ fil du temps, cette offre d'abord axée sur la découverte contemplative des géotopes a évolué vers l'amélioration des outils de médiation élaborés, participant ainsi de l'éducation à l'environnement, mais également vers la création de produits touristiques qui donnent à vivre une expérience au visiteur. Le géotourisme s'appuie donc sur des ressources patrimoniales qui lui sont propres et sur lesquelles se construisent des outils de valorisation qui empruntent de nombreuses pratiques au tourisme de découverte. Une implication plus directe des géoscientifiques dans ces processus de médiation devrait permettre la création d'outils spécifiques à ce champ disciplinaire qui manipule certains concepts qui lui sont propres : la durée des temps géologiques, la triple histoire des paysages (Marthaler, 2004) etc.

L'offre abondante, en expansion constante depuis une vingtaine d'années, ne simplifie pas son usage par les touristes. L'information est dispersée, difficilement accessible et très peu organisée en réseau si ce n'est celui du projet européen «Iron route» qui fédère plusieurs anciens sites miniers à travers les Alpes ou bien certaines associations professionnelles comme, en Suisse, l'ASECAT (Association Suisse des Exploitants de Cavernes Aménagées pour le Tourisme) qui fait la promotion de l'ensemble des grottes aménagées du pays. De plus, une grande majorité de l'offre concerne des sites d'importance locale pour lesquelles il est souvent difficile de connaître à l'avance l'existence d'un sentier géodidactique ou d'un écomusée sur le thème des géosciences.

Malgré tout, à l'échelle d'un territoire comme le massif alpin, en ne prenant en compte que les géosites touristiques pour lesquels il est possible d'obtenir des chiffres de fréquentation, ce sont plus de cinq millions de touristes (Cayla, 2009) qui chaque année visitent un lieu ayant un rapport avec la géologie. Le label «European Geopark», attribué à ce jour 
à quatre territoires alpins, est un moyen de faire connaître à l'échelle internationale les caractéristiques géotouristiques d'un territoire. Il pourrait être intéressant d'aller plus loin. En effet, chaque site géotouristique des Alpes éclairant un aspect de l'histoire anté-alpine, de l'orogenèse alpine, ou bien illustrant des événements géologiques, paléontologiques, géomorphologique de portée locale ou plus globale, leur mise en réseau au moyen de routes thématiques permettrait de les inscrire dans une histoire commune, celle de l'histoire géologique d'une chaîne de montagne. L'Année internationale de la planète Terre a permis de montrer l'exemple en instaurant la création, le long du parcours de la Via Alpina (itinéraire de randonnée qui traverse les Alpes depuis la Méditerranée jusqu'à Vienne), d'une Via Geoalpina qui permet la valorisation des géosciences tout au long de ce parcours (voir : http://www.viageoalpina.org).

\section{Références}

ALCIATI, Luca (2008a) « Roches ornementales des vallées de l'Ossola :

«Serizzo» et «Beola»», Pierre actual, n 858, p. 60-64.

ALCIATI, Luca (2008b) «Roches ornementales des vallées de l'Ossola : les granits», Pierre actual, $\mathrm{n}^{\circ} 861$, p. 86-92.

ALCIATI, Luca (2008c) «Les marbres de la vallée de l'Ossola», Pierre actual, $\mathrm{n}^{\circ}$ 864, p. 103-107.

BERREBI, Yaelle (2006) «Les sentiers didactiques. Analyse de la perception du public face à quatre réalisations géodidactiques ». Mémoire de licence, Unil : Institut de Géographie. 200 p.

BIOT, Vincent (2006) «Le tourisme souterrain en France», Karstologia Mémoire, $\mathrm{n}^{\circ} 15,236 \mathrm{p}$.

BOUÉ, Ami (1835) Guide du géologue-voyageur, t. 1, s. 1. : Levrault F.G. 594 p. BOUÉ, Ami (1836) Guide du géologue-voyageur, t. 2, s. l. : Levrault F.G. 594 p.

BUSOLINI, Patrizia (2007) « Reconverted mining sites/museums at European level. Networking Results», III International Conference on the Iron Route Huttenberg.

CAYLA, Nathalie (2009) «Le patrimoine géologique alpin : de la médiation à la valorisation géotouristique». Thèse de doctorat de sciences de la Terre, de l'univers et de l'environnement, Savoie : Université de Savoie. 309 p.

CHAPRON, Emmanuelle (2008) L'Europe à Nîmes : les carnets de JeanFrançois Séguier (1732-1783), s. 1. :A. Barthélémy. 205 p.

CIGNA, Arrigo A. et Erio BURRI (2000) «Development, management and economy of show caves", International journal of Speleology, vol. 29(B), p. 1-27.

DESHAIES, Michel (2007) Les territoires miniers Exploitation et reconquête, Coll. «Carrefours», s. 1. : Ellipse. 223 p.

DUVAL, Mélanie (2007) «Dynamiques spatiales et enjeux territoriaux des processus de patrimonialisation et de développement touristique. Étude comparée des gorges de l'Ardèche et du Karst slovène». Thèse de doctorat de géographie, Savoie : Université de Savoie. 514 p.

EDER, Renate (2007) «Geologie am weg - Anregungen zur Gestaltung von geotrails». Abhanlungen der geologischen Bundesanstalt, Helft 51, p. 29-34.

FILLIPELO, Andrea (2004) I lapidei ornamentali della provincia del VerbanoCusio-Ossola : Realizzazione di una banca dati geotematica. Facoltà di Scienza M.F.N. : Torino. 225 p.

FORTEY, Richard (2004) The earth, an intimate history, s. 1. : Harpers Collins publishers. $501 \mathrm{p}$.

FRANKS, Sibylle et Rudolf TRÜMPY (2005) «The Sixth International Geological Congress : Zürich, $1894 »$ Episodes, vol. 28, nº 3, p. 187-192.
GAUCHON, Christophe (1997) «Des cavernes et des Hommes - Géographie souterraine des montagnes françaises», Karstologia Mémoire, nº 7, 248 p.

GAUDANT, Jean (1997) «Les poissons pétrifiés du Monte Bolca (Italie) et leur influence sur les théories de la Terre au milieu du Siècle des lumières, d'après un manuscrit inachevé de Jean-Francois Seguier (17031784) », Bulletin de la Société Géologique de France; septembre, vol. 168. $\mathrm{n}^{\circ} 5$, p. 675-683.

GOHAU, Gabriel (2000) «Les scientifiques et «l'usage des montagnes» au XVIII siècle», DANS Une cordée originale: histoire des relations entre science et montagne?, Jean-Claude PONT et Jan LACKI (dir.), p. 80-95. Genève : Georg.

HOBLÉA, Fabien (2008) «La protection et la valorisation des patrimoines du karst», Géosciences, n 7-8, p. 101.

HOSE, Thomas A. (2008) «Towards a history of geotourism : definitions, antecedents and the future», The history of geoconservation, C. V. BUREK et C. D. PROSSER (éd.), p. 37-60.

JACOBI, Daniel (1999) La communication scientifique : discours, figures, modèles, coll. «Médias \& Sociétés», Grenoble : Presses Universitaires de Grenoble. 277 p.

KESTLER, Franz Xaver (2005) Der Tölzer Lobus des würmeiszeitlichen Isar-Loisach-Gletschers als Gegenstand einer geodidaktischen Exkursion. Fakultät für Geowissenschaften der Ludwig-Maximilians. 260 p.

MAISCH, Max; Conradin A. BURGA et Peter FITZE (1999) Lebendiges Gletschervorfeld, s. 1. : Engadin Press AG. 138 p.

MARTHALER, Michel (2004) «Lecture et analyse d'un paysage : Zermatt et le Cervin. Un exemple de la mémoire de la terre révélée par les panoramas», DANS Paysages géomorphologiques, E. REYNARD, J.-P. PRALONG (éd.), p. 51-66. Lausanne : Institut de Géographie («Travaux et Recherches", n 27).

PALME, Rudolf et Wolfgang INGENHAEFF (1993) Galeries, puits et minerais : À propos de l'histoire de l'industrie minière de Schwaz, s. 1. : Berenkamp. 108 p.

PHILIPPE, Michel; Alain ARGANT et Jacqueline ARGANT (2004) L'ours des cavernes: Actes du $9^{\circ}$ symposium international sur l'ours des cavernes: Entremont-le-Vieux (Savoie : 25-27 septembre 2003). 208 p.

PRALONG, Jean-Pierre (2006) «Géotourisme et utilisation de sites naturels d'intérêt pour les sciences de la Terre : Les régions de Crans-MontanaSierre (Valais, Alpes suisses) et Chamonix-Mont-Blanc (Haute-Savoie, Alpes françaises) ». Thèse de doctorat de la faculté des géosciences et de l'environnement, Lausanne : Université de Lausanne. 223 p.

RASSE, Paul (1999) Les musées à la lumière de l'espace public Histoire, évolution, enjeux. Paris : L'Harmattan. 240 p.

ROHN-BROSSARD, M. (2006) «Parc naturel, chemin à thème et impact économique». Travail de diplôme de formation continue en écologie et en sciences de l'environnement, Neufchâtel : Université de Neuchâtel. $91 \mathrm{p}$.

SAND, Georges (1839) Lettre d’un voyageur, t. 1, Paris : Félix Bonnaire.

SELLIER, Dominique (2009) «La vulgarisation du patrimoine géomorphologique : objets, moyens et perspectives ", Bulletin de l'Association de Géographes français, n 1, p. 67-81.

UNESCO (2005) «UNESCO: World Heritage Convention », $29^{\text {th }}$ session of the Committee: WHC-05/29.COM/INF.8B.2, <http://whc.unesco.org/ en/sessions/29COM/documents/>, consulté le 23 novembre 2010.

VACCARI, Ezio (2003) «Mais qui a donc enfanté l'alpe ?», L’Alpe, vol. 20, p. 82-89.

ZHANG, Shouyne et Yuzhang JIN (1994) Tourism resources on karst \& caves in China : $2^{e}$ Congrès de l'ISCA (Malaga : 29 octobre au $1^{\text {er }}$ novembre 1994), p. 111-119. 